\title{
APLICAÇÃO DE LASER EM LESÕES BUCAIS DE PÊNFIGO VULGAR - RELATO DE CASO CLÍNICO
}

\author{
LASER APPLICATION IN PEMPHIGUS VULGARIS ORAL INJURIES - \\ CLINICAL CASE REPORT
}

Karina e Silva Pereira ${ }^{1}$.

\begin{abstract}
RESUMO
Introdução: O pênfigo vulgar é uma doença autoimune e rara, que geralmente acomete indivíduos de meia idade. As lesões orais do pênfigo vulgar normalmente são os primeiros sinais da doença caracterizadas clinicamente como múltiplas e extensas ulcerações, sendo bastante dolorosas, provocando dificuldade de mastigação, fonação e deglutição. O laser de baixa intensidade é um método não invasivo, eficaz e de baixo custo, sendo utilizado com o objetivo de diminuir a dor na cavidade oral e estimular a cicatrização das lesões, facilitando a ingestão de alimentos e a higiene oral adequada. Desenvolvimento: Paciente, 41 anos, gênero feminino, foi atendida no Hospital de Doenças Tropicais do Tocantins da Universidade Federal do Tocantins (HDT-UFT) com relato de perda de peso (+- $9 \mathrm{Kg}$ ) e lesões em cavidade oral há cerca de 3 meses. Após avaliação dermatológica e odontológica, constatada a presença de lesões em pele e mucosas características de lesões por doença autoimune foi realizada a manobra para constatação do sinal de Nikolsky, apresentando sinal positivo e com a confirmação do exame histopatológico foi fechado o diagnóstico de pênfigo vulgar. Foram realizadas três aplicações de laser sobre as lesões da cavidade oral, e após oito dias de internação a paciente evoluiu com melhora do aspecto clínico destas, redução da dor, ingestão de alimentos mais sólidos e ganho de peso, recebendo alta hospitalar em bom estado geral. Considerações Finais: O presente caso clínico demonstra a importância da atuação multidisciplinar para o estabelecimento diagnóstico precoce de doenças com manifestações bucais como o pênfigo vulgar, favorecendo o estabelecimento do tratamento e melhorando o prognóstico do paciente. Ademais, a aplicação do laser de baixa intensidade foi extremamente importante no que tange à redução da sintomatologia dolorosa e cicatrização das lesões orais, pois demonstrou eficácia a partir da primeira sessão, contribuindo para o bem-estar geral da paciente.
\end{abstract}

Citação: Pereira KS. (2021) Aplicação de laser em lesões bucais de pênfigo vulgar relato de caso clínico. Revista de Patologia do Tocantins, 8(2).

Instituição: ${ }^{1}$ Cirurgiã Dentista Especialista em Implantodontia e Especialista em Odontologia do Trabalho. Hospital de Doenças Tropicais do Tocantins da Universidade Federal do Tocantins (HDTUFT). Araguaína-TO, Brasil

Autor correspondente: Karina e Silva Pereira. Rua das Quineiras, Qd. L22, Lt. 32, residencial Topázio, Araguaína-TO esilvakarina@yahoo.com

Editor: Rosa A. C. G. Medicina, Universidade Federal do Tocantins, Brasil.

Publicado: 25 de julho de 2021.

Direitos Autorais: (c) 2021 Pereira et al. Este é um artigo de acesso aberto que permite o uso, a distribuição e a reprodução sem restrições em qualquer meio, desde que o autor original e a fonte sejam creditados.

Conflito de interesses: os autores declararam que não existem conflitos de interesses.

\begin{abstract}
Introduction: Pemphigus vulgaris is a rare autoimmune disease that usually affects middleaged individuals. Oral lesions of pemphigus vulgaris are usually the first signs of the disease. They present clinically as multiple and extensive ulcerations, covered by a pseudomembrane, being quite painful, causing difficulty in chewing, phonation and swallowing. The low intensity laser presents itself as an aid in the treatment of ulcerated lesions. It is a noninvasive, effective and inexpensive method, being used to reduce pain in the oral cavity and stimulate wound healing, facilitating food intake and proper oral hygiene, increasing the patient's quality of life. Development: A 41-year-old female patient was admitted to the Tocantins Tropical Diseases Hospital of the Federal University of Tocantins (HDT-UFT) with weight loss (+ - $9 \mathrm{Kg}$ ). ) and lesions in the oral cavity about 3 months. After dermatological evaluation, together with the dental evaluation, found the presence of lesions on the skin and mucous membranes characteristic of lesions due to autoimmune disease, the maneuver was performed to verify the Nikolsky sign, presenting positive sign and the result of the histopathological examination was closed. diagnosis of pemphigus vulgaris. Three laser applications were performed on the oral cavity lesions, and after eight days of hospitalization the patient evolved with an improvement in the clinical aspect of oral lesions, pain reduction, more solid food intake and weight gain, being discharged in good hospital. general state. Final Considerations: This case report demonstrates the importance of multidisciplinary action for the early diagnosis of diseases with oral manifestations such as pemphigus vulgaris, favoring the establishment of treatment and improving the patient's prognosis. In addition, the application of low intensity laser was extremely important regarding the reduction of painful symptoms and healing of oral lesions, as it demonstrated efficacy from the first session, contributing to the patient's general well-being.

key-words: Pemphigus vulgaris; Laser; Oral lesions.
\end{abstract}




\section{INTRODUÇÃO}

O pênfigo representa um grupo de doenças autoimunes, raras, que se caracterizam pela presença de lesões bolhosas e ulceradas em pele e mucosas. Existem quatro subtipos de pênfigo: vulgar, vegetante, eritematoso e foliáceo. O pênfigo vulgar é o tipo que apresenta maior prevalência de acometimento da mucosa oral, sendo comum em indivíduos de meia idade ${ }^{1}$.

As lesões orais do pênfigo vulgar normalmente são os primeiros sinais da doença, antecedendo as lesões cutâneas. Clinicamente apresenta-se como múltiplas e extensas ulcerações, cobertas por uma pseudomembrana, antecedidas por lesões bolhosas. Os locais mais acometidos são a mucosa jugal, língua e gengiva. As lesões são bastante dolorosas, tendo como consequência dificuldade de mastigação, fonação e deglutição. Frequentemente ocorrem lesões em pele e em outras mucosas².

O tratamento tópico das lesões orais está indicado quando são observadas lesões localizadas e brandas, podendo ser utilizado o Propionato de Clobetasol (0,05\%) em gel ou elixir, bochechos de Betametasona elixir $(0,1 \mathrm{mg} / \mathrm{ml})$ e Triancinolona gel. Quando estão presentes múltiplas lesões em diversos locais da cavidade oral e/ou tem a presença de lesões em outras regiões do corpo, a associação com corticoide sistêmico está indicada ${ }^{3}$.

O uso frequente de corticoides sistêmicos, porém, pode induzir diversas alterações, tais como: diabetes melito, osteoporose, alterações no humor, ulcerações no trato gastrointestinal, artrite reumatoide, doença autoimune da tireoide e miastenia grave, sendo necessária a avaliação periódica do paciente ${ }^{4}$.

O laser de baixa intensidade é um método não invasivo, eficaz e de baixo custo, sendo utilizado com o objetivo de diminuir a dor na cavidade oral - causada pelas lesões ulceradas - e estimular a cicatrização das lesões, facilitando a ingestão de alimentos e a higiene oral adequada, elevando a qualidade de vida do paciente ${ }^{5}$.

\section{DESCRIÇÃO DO CASO}

Paciente M.D.S.S., 41 anos, gênero feminino, foi admitida no Hospital de Doenças Tropicais do Tocantins da Universidade Federal do Tocantins (HDT-UFT) proveniente da Unidade de Pronto Atendimento (UPA) de Araguaína com com relato de perda de peso (+- $9 \mathrm{Kg}$ ) e lesões em cavidade oral já cerca de 3 meses. O encaminhamento ao hospital ocorreu devido a hipótese diagnóstica inicial ser de paracoccidioidomicose.

Ao exame clínico a paciente apresentava-se consciente, orientada, emagrecida, desidratada, com abdome escavado e com a presença de disfagia em consequência das lesões em orofaringe. $\mathrm{O}$ exame clínico odontológico constatou a presença de diversas lesões ulceradas em língua, mucosas, lábios, palato e faringe como demonstram as figuras 01,02 e 03; a paciente ainda apresentava sialorréia e má higiene oral decorrente da presença das lesões. A paciente negou ter alergia a qualquer substância e quando questionada, relatou a ocorrência de bolhas na pele. As hipóteses diagnósticas foram então: estomatite aftosa, pênfigo vulgar e paracoccidioidomicose.
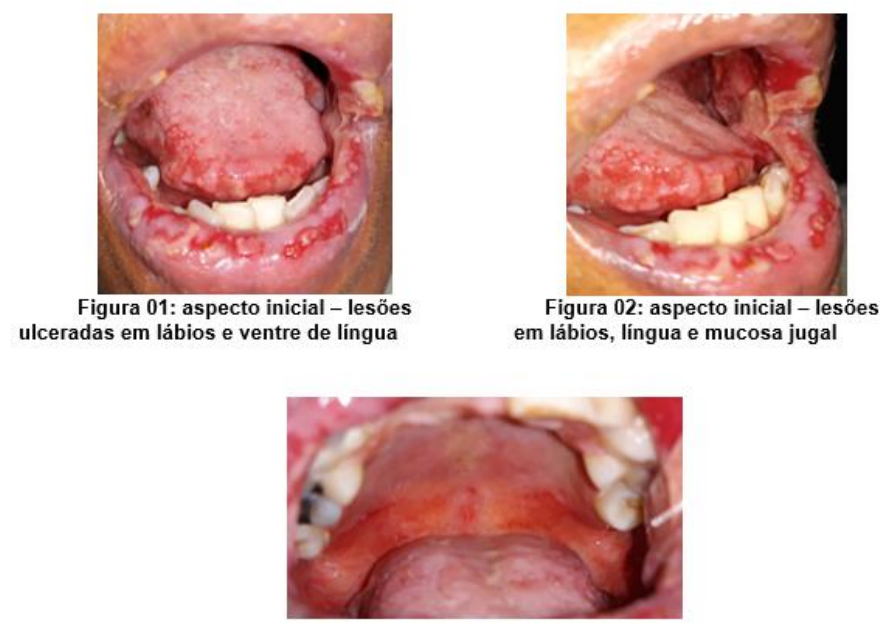

Figura 03: aspecto inicial - lesão em palato mole

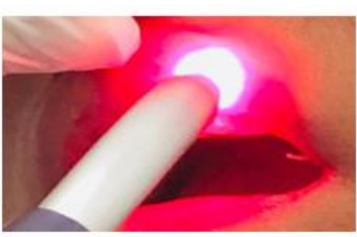

Figura 04: aplicação de laser de baixa intensidade em lábio

A conduta inicial foi baseada no alívio da sintomatologia dolorosa e estabelecimento diagnóstico. A paciente foi orientada em como realizar a higienização oral na condição atual, utilizando escova dentária infantil, creme dental fluoretado e digluconato de clorexidina $0,12 \%$ para bochecho duas vezes ao dia como complementação à escovação, reduzindo o número de bactérias orais e evitando infecção secundária. Foi estabelecida dieta pastosa devido a dificuldade de mastigação e deglutição.

Para a redução da dor foi estabelecido a aplicação de laser de baixa intensidade vermelho $2 \mathrm{~J} / \mathrm{cm}^{2}$ (figura 04 ) em 23 pontos da cavidade oral distribuídos entre língua, lábios, mucosa jugal e palato mole.

Foram realizadas coletas para exames laboratoriais, pesquisa de BAAR, bem como radiografia de tórax e biópsia de lesão oral para confirmação diagnóstica.

Os exames laboratoriais - hemograma, glicose, uréia, creatinina, TGO, TGP, fosfatase alcalina, bilirrubina, gama GT, cálcio, sódio, potássio, proteínas totais e frações - não apresentaram valores alterados. A pesquisa de BAAR foi negativa. A radiografia de tórax não demonstrou alterações significativas, o diagnóstico de paracoccidioidomicose foi descartado.

Após avaliação dermatológica, em conjunto com a avaliação odontológica, constatada a presença de lesões em pele e mucosas características de lesões por doença autoimune, realizada a manobra para constatação do sinal de Nikolsky, apresentando sinal positivo e com o resultado do exame histopatológico foi fechado o diagnóstico de pênfigo vulgar. 
Foram realizadas três aplicações de laser sobre as lesões da cavidade oral, e após oito dias de internação a paciente evoluiu com melhora do aspecto clínico das lesões orais, redução da dor, ingestão de alimentos mais sólidos e ganho de peso, recebendo alta hospitalar em bom estado geral (Figuras 05, 06, 07, 08 e 09).
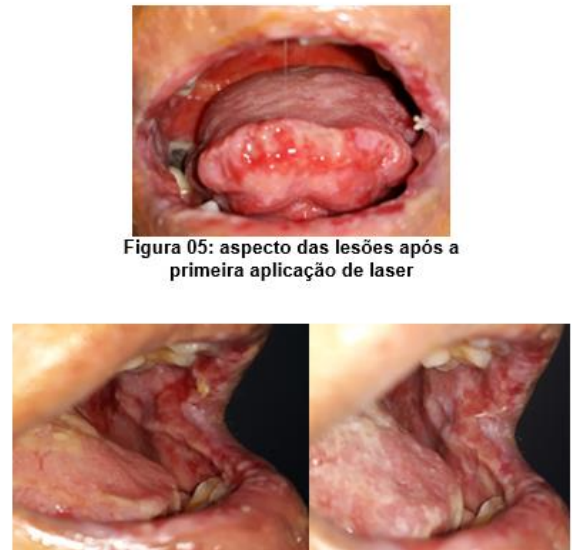

Figuras 06 e 07 : aspecto das lesões orais após a segunda aplicação de laser

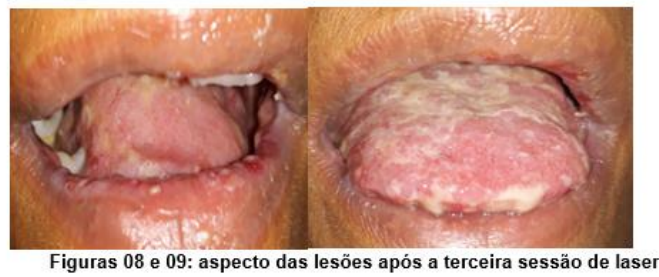

\section{DISCUSSÃO}

As lesões de pênfigo vulgar se desenvolvem em virtude de uma alteração no sistema imunológico do paciente, no qual ocorre a produção de anticorpos contra a desmogleína 1 e 3, proteínas encontradas nos desmosomos que auxiliam na adesão das células epiteliais ${ }^{2}$.

O diagnóstico clínico do pênfigo vulgar deve ser confirmado com o exame histopatológico e em alguns casos complementado com a imunofluorescência. Um elemento que também pode auxiliar no diagnóstico é a verificação de positividade do sinal de Nikolsky, manobra semiotécnica que consiste no desprendimento das camadas superficiais da mucosa das camadas inferiores com uma ligeira fricção, expondo-se o tecido conjuntivo e formando-se uma úlcera ${ }^{6}$. 0 presente caso foi diagnosticado clinicamente por cirurgiã dentista e dermatologista com base nas características clínicas das lesões, apresentando sinal de Nikolsky positivo na mucosa oral, sendo confirmado o diagnóstico por exame histopatológico.

O pênfigo vulgar é uma doença de prognóstico sombrio, apresentando alta taxa de mortalidade em casos não tratados. O diagnóstico e tratamento desta doença são de origem multidisciplinar ${ }^{1}$. Neste estudo, a paciente foi avaliada e acompanhada por cirurgiã dentista, médicos (clínico geral, dermatologista e clínico geral), além de nutricionista e equipe de enfermagem. Sendo o trabalho multidisciplinar extremamente importante para a recuperação da paciente.

Gonçalo (2016) afirma que as lesões orais devem ser tratadas de forma tópica quando brandas e quando há ocorrência de lesões múltiplas e mais graves a associação de corticoides sistêmicos deve ser feita. Já Ferreira et al. (2016) afirmam que o tratamento de escolha deve ser o uso de corticoides sistêmicos com ou sem associação de drogas imunorreguladoras, utilizando-se altas doses do medicamento inicialmente e reduzindo-se gradativamente até a resolução das lesões, pois verificaram em seu estudo que mesmo utilizando a medicação tópica em forma de bochecho por três semanas o paciente não apresentou melhora significativa.

Lesões orais ulceradas provocam intensa sensibilidade dolorosa e podem permanecer por tempo prolongado, caso não haja intervenção. O laser de baixa potência é capaz de estimular a produção de ATP pela mitocôndria, conferindo propriedades terapêuticas importantes como a biomodulação dos tecidos afetados, além da ação analgésica e antiinflamatória, sendo fundamental para remissão completa das lesões ullceradas, inclusive possibilitando a alimentação do paciente $\mathrm{e}$ melhorando sua qualidade de vida ${ }^{5}$.

Neste caso relatado, a aplicação de laser de baixa intensidade foi eficaz ao reduzir a sintomatologia dolorosa a partir da primeira sessão e promover a cicatrização das lesões orais mais rapidamente, possibilitando uma melhora na higienização oral e na alimentação, que passou de pastosa para branda após três sessões de aplicação do laser, contribuindo assim para a recuperação do peso corporal da paciente.

No estudo de Gonçalo (2016), a terapia com laser de baixa intensidade foi utilizada em pacientes com pênfigo vulgar, sendo considerada eficaz por reduzir a sintomatologia e o número de lesões orais, não reduzindo porém a recorrência das mesmas.

Minicucci et al. (2006) observaram em seu estudo melhora e diminuição no número de lesões após 7 a 10 sessões de laserterapia, sendo relatada pelos pacientes a redução de cerca de $70 \%$ da dor após a primeira sessão e sua total remissão após a terceira sessão.

\section{CONSIDERAÇÕES FINAIS}

O presente caso clínico demonstra a importância da atuação multidisciplinar para o estabelecimento diagnóstico precoce de doenças com manifestações bucais como o pênfigo vulgar, favorecendo o estabelecimento do tratamento e melhorando o prognóstico do paciente.

Ademais, a aplicação do laser de baixa intensidade foi extremamente importante no que tange à redução da sintomatologia dolorosa e cicatrização das lesões orais, pois demonstrou eficácia a partir da primeira sessão, contribuindo para o bem estar geral da paciente.

O laser de baixa intensidade aplicado sobre lesões de pênfigo vulgar deve ser estudado de forma mais abrangente, pois apresenta um grande potencial como coadjuvante do tratamento sistêmico, podendo talvez auxiliar para a redução da dose e tempo de uso de corticoides e na diminuição consequente de seus efeitos colaterais. 


\section{REFERÊNCIAS BIBLIOGRÁFICAS}

1. Miziara ID, Ximenes Filho JA, Ribeiro FC, et al. Acometimento oral no Acometimento oral no pênfigo vulgar. Rev. Bras. Otorrinolaringol.v.69, n.3, 327-31, mai./jun. 2003

2. Gonçalo RIC, Severo MLB, Medeiros AMC, et al. Vesiculobullous autoimmune diseases with oral mucosa manifestations: retrospective and follow-up study. RGO, Rev Gaúch Odontol. 2018 Jan-Mar; 66(1):42-49

3. Gonçalo RIC. Doenças Autoimunes Bolhosas com Manifestação em Mucosa Oral: Estudo Retrospectivo e de Acompanhamento. 2016. Monografia (graduação em Odontologia). Universidade Federal do Rio Grande do Norte, Natal-RN. 38p

4. Wormser D, Chen DM, Sun BD, et al. Cumulative oral corticosteroid use

5. increases risk of glucocorticoid-related adverse events in patients with newly diagnosed pemphigus. J AM ACAD DERMATOL. v. 77, n. 2. p 379-381. 2017

6. Gomes DAC, Gomes EAC, Picciani BLS, et al. Aplicações da laserterapia no tratamento de lesões orais ulceradas. Revista Brasileira de Odontologia. 2019; 76 (Supl 2): 68

7. Risso $M$, Villalpando $K T$, Pinho $M N$, et al. Pênfigo vulgar: relato de caso clínico. RGO - Rev Gaúcha Odontol., Porto Alegre, v.59, n.3, p.515-520, jul./set., 2011

8. Ferreira VYN, Lyra TC, Pereira LL, et al. Eficácia do uso de corticosteroide sistêmico no tratamento do pênfigo vulgar oral. Arq. Ciênc. Saúde. 2016 jul-set; 23(3) 10-13

9. Piacentini M. Condutas Clínicas Frente ao Paciente Portador de Doenças Autoimunes na Cavidade Bucal. 2013. Monografia (graduação em Odontologia). Universidade Federal de Santa Catarina, Florianópolis. 58p.

10. MINICUCCI E, BARRAVIERA S, LOPES AL.Low-level laser therapy on the treatment of oral and cutaneous pemphigus vulgaris: case report.Lasers Medicine Science, London. v.27. p.1103-1106. 2006. 